\title{
Tools for Multimode Quantum Information: Modulation, Detection, and Spatial
} Quantum Correlations

Lassen, Mikael Østergaard; Delaubert, Vincent; Janousek, Jirí; Wagner, Kate; Bachor, Hans. A-; Lam, Ping Koy; Treps, Nikola; Buchhave, Preben; Fabre, Claude; Harb, Charles

Published in:

Physical Review Letters

Link to article, DOI:

10.1103/PhysRevLett.98.083602

Publication date:

2007

Document Version

Publisher's PDF, also known as Version of record

Link back to DTU Orbit

Citation $(A P A)$ :

Lassen, M. Ø., Delaubert, V., Janousek, J., Wagner, K., Bachor, H. A., Lam, P. K., Treps, N., Buchhave, P., Fabre, C., \& Harb, C. (2007). Tools for Multimode Quantum Information: Modulation, Detection, and Spatial Quantum Correlations. Physical Review Letters, 98(8), 083602. https://doi.org/10.1103/PhysRevLett.98.083602

\section{General rights}

Copyright and moral rights for the publications made accessible in the public portal are retained by the authors and/or other copyright owners and it is a condition of accessing publications that users recognise and abide by the legal requirements associated with these rights.

- Users may download and print one copy of any publication from the public portal for the purpose of private study or research.

- You may not further distribute the material or use it for any profit-making activity or commercial gain

- You may freely distribute the URL identifying the publication in the public portal 


\title{
Tools for Multimode Quantum Information: Modulation, Detection, and Spatial Quantum Correlations
}

\author{
M. Lassen, ${ }^{1,2}$ V. Delaubert, ${ }^{1,3}$ J. Janousek,${ }^{1,2}$ K. Wagner, ${ }^{1}$ H.-A. Bachor, ${ }^{1}$ P. K. Lam, ${ }^{1}$ N. Treps,${ }^{2}$ \\ P. Buchhave, ${ }^{2}$ C. Fabre, ${ }^{3}$ and C. C. Harb ${ }^{4,1, *}$ \\ ${ }^{1}$ Australian Center for Quantum-Atom Optics, The Australian National University, Canberra ACT 0200, Australia \\ ${ }^{2}$ Department of Physics, DTU, Building 309, 2800 Lyngby, Denmark \\ ${ }^{3}$ Laboratoire Kastler Brossel, 4 place Jussieu, case 74, Paris 75252 Cedex 05, France \\ ${ }^{4}$ School of Information Technology and Electrical Engineering, University College, The University of New South Wales, \\ Canberra, ACT, 2600 \\ (Received 14 July 2006; revised manuscript received 5 December 2006; published 22 February 2007)
}

\begin{abstract}
We present the key elements required for continuous variable parallel quantum information protocols based on spatial multimode quantum correlations. We describe techniques for encoding, combining and detecting spatial quantum information with high efficiency in the individual transverse modes. Until now, the missing feature for the implementation of such protocols was the generation of squeezing in higher order transverse Hermite-Gauss modes. We experimentally demonstrate squeezing in selective modes by fine-tuning the phase matching condition of the nonlinear $\chi^{(2)}$ material and the cavity resonance condition of an optical parametric amplifier. Combined, these results open the way to practical multimode optical quantum information systems.
\end{abstract}

DOI: 10.1103/PhysRevLett.98.083602

PACS numbers: 42.50.Dv, 03.67.-a

Optical quantum communication and information processing is developing rapidly [1]. Both single photon and continuous variables $(\mathrm{CV})$ are being used, already enabling the generation, transmission, and detection of quantum correlations. CV optical techniques include close to perfect modulation and detection schemes, which is a requirement for the effective use of squeezed and entangled light [2-5] for applications such as sensing and gravitational-wave detection [6,7], quantum communication and teleportation [8], dense coding [9], secret sharing [10], noiseless amplification [11], and possibly quantum logic [12].

So far, CV techniques typically use a single mode and a pair of conjugate quantum parameters per beam: mean field amplitude and phase quadratures or polarization parameters. Using this set of variables, several key elements are required to implement quantum information protocols. The first one is the efficient signal encoding of the individual conjugate observables, and is typically achieved with electro-optic modulation (EOM) of the sidebands at frequency $\Omega$ around the central laser frequency $\nu_{L}$. These sidebands can be used to carry the information. Second, CV schemes use high efficiency phase sensitive detectors, such as homodyne detectors, that allow the interrogation of each quadrature individually. Finally, they require squeezed light, i.e., pairs of quantum correlated sidebands at frequency $\nu_{L} \pm \Omega$, allowing performances with signal to noise ratio better than the conventional quantum noise limit (QNL), and the generation of quadrature or polarization entanglement [13].

CV experiments have traditionally been performed with $\mathrm{TEM}_{00}$ profile for the squeezed beam and the homodyne detection local oscillator (LO). At the same time, parallel research efforts into $\mathrm{CV}$ quantum imaging have been de- veloped [14], and concentrated on the transmission, amplification, and processing of spatial information with quantum correlations $[15,16]$. Recent results include the detection of spatial parameters such as small displacements below the QNL [17], the optimization of imaging techniques [18], and proposals for more advanced applications such as super-resolution [19] and quantum holography [20].

The aim of our research is to use the spatial properties of light to demonstrate that one optical beam can convey several independent channels of quantum information. This can be used for quantum communication or spatial sensing, as suggested in Figs. 1(a) and 1(b), respectively. Such multimode systems have advantages in regard to the complexity of the protocols [21]. This work with continuous variables complements the single photon systems, designed to transmit not just qubits but qudits, which use photon states with different angular momenta [22,23].

Here we present all the key elements required for extending CV quantum optics from the single mode case to a multimode system. The quantum channels are provided by the TEM $_{n m}$ Hermite-Gauss (H-G) modes. The orthogonality of these modes allows us to address the parallel channels individually and to combine them with minimal loss and negligible crosstalk. Second, we report more than $90 \%$ detection efficiencies of the individual channels. Finally, we describe how to efficiently generate spatially squeezed light in higher order H-G modes. Together these achievements provide a complete multimode quantum information system and demonstrate its efficiency.

For our investigation, we focus on the lowest order $\mathrm{H}-\mathrm{G}$ modes: $\mathrm{TEM}_{00}, \mathrm{TEM}_{10}$, and $\mathrm{TEM}_{20}$. These modes are directly related to small spatial variations of a Gaussian 

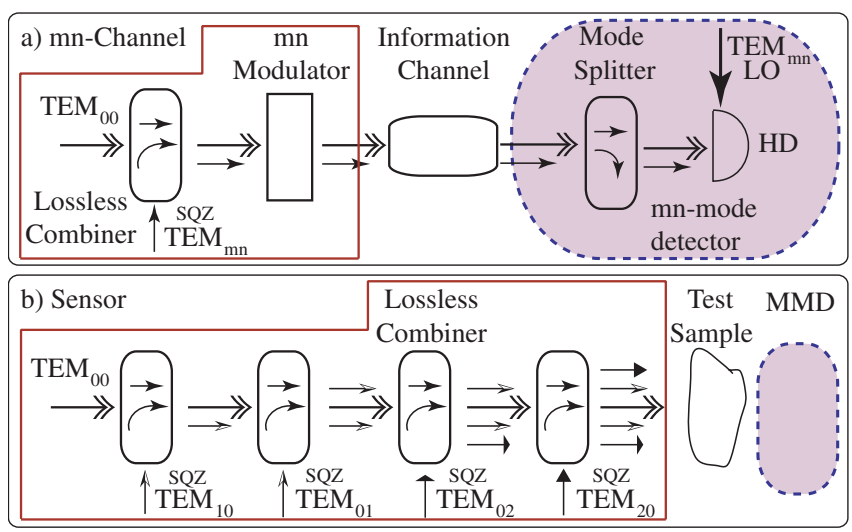

FIG. 1 (color online). Practical spatial multimode systems: (a) single quantum information channel including modulation, propagation, and detection scheme in the case of information encoded on a $\mathrm{TEM}_{m n}$ mode; (b) measurement of the spatial properties of a test sample using lossless combiners and multimode detectors (MMD). SQZ: squeezed. HD: homodyne detection.

beam [24]. In particular, the real and imaginary parts of the $\mathrm{TEM}_{10}$ mode represent changes in transverse position $\delta d$ and tilt $\delta \theta$ of a $\mathrm{TEM}_{00}$ beam [25], whereas the real and imaginary parts of the TEM 20 mode are related to a waist size mismatch $\delta w$ and a waist position mismatch along the propagation axis $\delta z$. The following equation quantifies these relationships:

$$
\begin{aligned}
E(x)= & A_{0}\left[u_{0}(x)+\left(\frac{\delta d}{w_{0}}+i \frac{\pi w_{0} \delta \theta}{\lambda}\right) u_{1}(x)+\frac{\delta w}{\sqrt{2} w_{0}} u_{2}(x)\right. \\
& \left.+i \frac{\delta z \lambda}{2 \sqrt{2} \pi w_{0}^{2}}\left(\frac{5}{\sqrt{2}} u_{0}(x)+u_{2}(x)\right)\right],
\end{aligned}
$$

where the spatial function $u_{n}(x)$ refers to the profile of the $\mathrm{TEM}_{n 0}$ mode. $A_{0}$ is the average amplitude, $w_{0}$ is the waist size, and $\lambda$ is the wavelength. Similar beam properties along the orthogonal transverse coordinate $y$ are carried by the $\mathrm{TEM}_{01}$ and $\mathrm{TEM}_{02}$ modes. We can encode information directly into the transverse orthogonal modes with close to perfect efficiency using simple electro-optic or mechanical spatial modulators acting on a $\mathrm{TEM}_{00}$ beam, such as a piezoelectric device, a tilted EOM, or a movable lens $[17,26]$.

For combining and analyzing orthogonal modes without loss, as schematically shown in Fig. 1(a), we can use a high finesse ring cavity, or alternatively a planar Mach Zehnder interferometer, parallel to $x$ or $y$, with an odd number of mirrors, in order to combine even and odd modes. We have demonstrated in our earlier work more than $95 \%$ efficiency for these components $[17,26]$. We thus have the ability to synthesize a multimode laser beam which combines the $\mathrm{TEM}_{00}$ mode with the higher order transverse modes $\mathrm{TEM}_{01}, \mathrm{TEM}_{10}, \mathrm{TEM}_{02}$, and $\mathrm{TEM}_{20}$. The resulting combination provides each channel with two quadratures of a conjugate pair of quantum variables, as shown in Fig. 1(a) in the case of a single channel carried by the $\mathrm{TEM}_{n m}$ mode. It is then straightforward to extend this scheme to several quantum channels. A similar scheme presented on Fig. 1(b) can be used as a sensor for spatial modification. Here a multimode squeezed beam is incident on a sample and detected with a multimode detector (MMD), which corresponds to cascaded single channel detectors presented in Fig. 1(a). Each channel provides information on a specific spatial property of the sample with sub-QNL precision.

The detection of these modes requires special attention. Conventional spatial detectors, such as split detectors or diode arrays, are simple but provide only limited efficiency. For example, the best theoretical efficiency of a split detector for displacement measurements is only $64 \%$ in intensity [25]. In contrast, with "spatial" homodyne detectors which use a LO beam that is mode matched to the specific $\mathrm{TEM}_{n m}$ channel, we reported more than $96 \%$ detection efficiency [26].

The crucial component missing until recently was the efficient generation of squeezing in the $\mathrm{TEM}_{n m}$ modes. We propose here to fill this technology gap by showing that the phase matching of a type I second order nonlinear process can be used to selectively choose the spatial profile of the squeezed mode, using an optical parametric amplifier (OPA). An OPA is a phase dependent device which, when operated below threshold, can reduce the noise in one quadrature to below the QNL (squeezing), while the conjugate quadrature shows extra noise (antisqueezing). This was first demonstrated by Wu et al. in 1986 [27]. Since then OPAs have reliably produced up to $-7 \mathrm{~dB}$ of squeezing $[28,29]$.

Producing squeezed light in any of the $\mathrm{TEM}_{n m}$ modes using an OPA requires the optimization of three conditions. First, the OPA resonator needs to be resonant for the H-G mode of interest. This is simply achieved by misaligning a $\mathrm{TEM}_{00}$ seed beam in order to couple light into the desired component and to lock the length of the cavity to its resonance. Second, the spatial profiles of the pump beam, at frequency $2 \nu_{L}$, and the seed beam, at frequency $\nu_{L}$, need to be chosen to maximize the spatial overlap and at the same time the nonlinear gain of the down-conversion process. Finally, phase matching has to be set to favor the conversion into the required spatial mode.

To understand how to optimize the last two conditions, let us now concentrate on the reverse process of optical parametric amplification, namely, second harmonic generation (SHG). When such systems are operated with higher order spatial modes, optimal phase matching occurs at different crystal temperatures, since each mode acquires different Gouy phase shift, when propagating through the nonlinear medium. As a demonstration of this effect, we pump a single pass SHG with a TEM $_{n 0}$ pump mode, as shown in the upper part of Fig. 2. We use a $\mathrm{MgO}_{\mathrm{LiNbO}}$ lithium niobate crystal, whose birefringence is highly tem- 

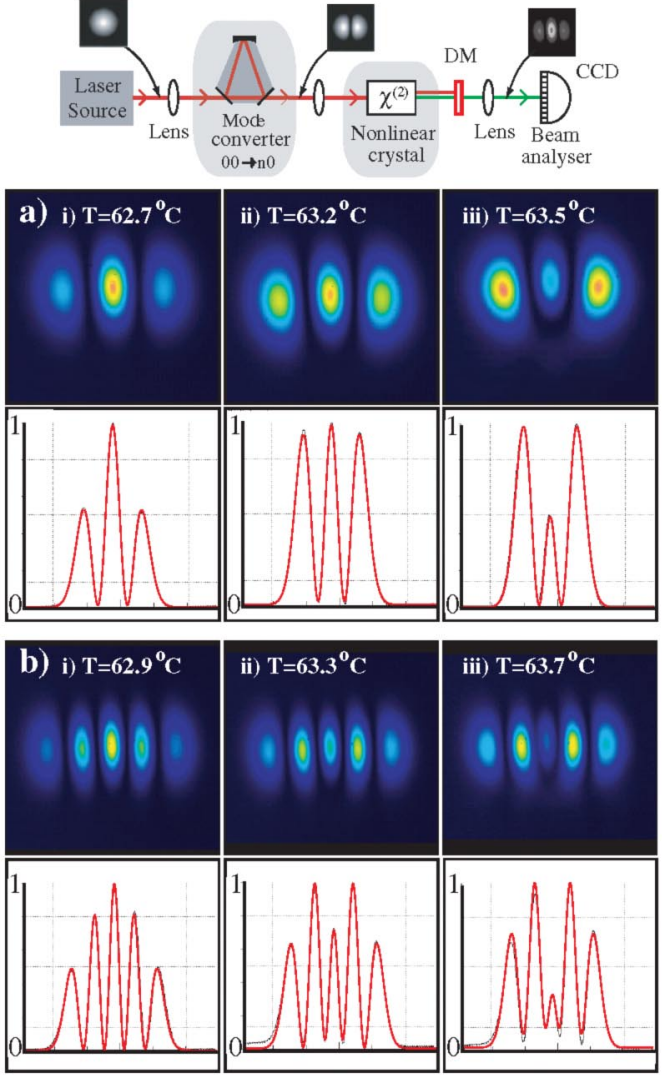

FIG. 2 (color). Top: Scheme for single pass SHG measurement in the case of $\mathrm{TEM}_{10}$ fundamental mode operation. Below: SH profiles generated in the crystal far field for three phase matching temperatures with a (a) $\mathrm{TEM}_{10}$ and (b) $\mathrm{TEM}_{20}$ pump profile. The cross-section traces show excellent between experimental results and theory fits.

perature dependent, and therefore allows adjustment of the phase matching condition for a particular spatial mode. The $\mathrm{TEM}_{n 0}$ pump mode, produced at $1064 \mathrm{~nm}$ by a diodepumped NPRO Nd:YAG laser, is generated by misaligning a ring cavity, designed to prevent any transverse mode degeneracy and locked to the resonance of the $\mathrm{TEM}_{n 0}$ mode. This mode converting device delivers a pure transverse $\mathrm{TEM}_{n 0}$ output mode, which is focused into the crystal such that $z_{R}=l / 2$, where $z_{R}$ is the beam Rayleigh range and $l$ is the crystal length. A dichroic mirror, DM, is used to filter out the fundamental pump field, and the second harmonic $(\mathrm{SH})$ profile is detected with a $\mathrm{CCD}$ camera in the far field.

The generated SH spatial profiles, normalized to their maximum, are presented for different crystal temperatures in Figs. 2(a) and 2(b) for TEM 10 and $\mathrm{TEM}_{20}$ pump modes, respectively. The spatial distribution of the $\mathrm{SH}$ field varies strongly with the crystal temperature. Tuning the temperature allows reproducible control over the conversion into other $\mathrm{TEM}_{n 0}$ modes. In the case of a $\mathrm{TEM}_{10}$ pump the SH field can be tuned from a predominantly $\mathrm{TEM}_{00}$ to a predominantly $\mathrm{TEM}_{20}$ profile, as shown in Fig. 2(a).
Similarly, for the $\mathrm{TEM}_{20}$ pump mode the SH field is a temperature dependent linear combination of the $\mathrm{TEM}_{00}$, $\mathrm{TEM}_{20}$, and $\mathrm{TEM}_{40}$ modes, as shown in Fig. 2(b). For a comparison of the experimental data with a quantitative model, we calculate the mode overlap between the $\mathrm{TEM}_{n 0}$ modes [30]. We predict the spatial intensity distribution of the SH light for the different temperatures, as shown in Fig. 2 in the lower diagrams together with the experimental profiles. They are in excellent agreement and show the tunability of the mode composition via crystal temperature.

We use this technique for the generation of squeezed light. As SHG and OPA operation are reverse processes, the SH beam profiles presented in Fig. 2 correspond to the optimal pump shape for OPA operation for a given crystal temperature. Optimal conversion efficiency is expected when the pump profile corresponds to the square of the fundamental. Although generating such a complicated beam to pump the OPA is possible by using holograms [31] or a spatial light modulator, at the cost of reduced parametric interaction, we choose to simply use the $\mathrm{TEM}_{00}$ mode delivered by our dual input laser at $532 \mathrm{~nm}$, and optimize the crystal temperature for this operation. A schematic diagram of our experimental setup to generate squeezing in higher order modes is presented in Fig. 3. All the technical details are presented in Ref. [30].

The measured squeezing in the three lowest order $\mathrm{H}-\mathrm{G}$ modes is shown in Fig. 4 with a resolution bandwidth of $300 \mathrm{kHz}$ and a video bandwidth of $300 \mathrm{~Hz}$. All traces are normalized to the QNL, given by trace (ii), which is measured by blocking the squeezed beam before the homodyne detector. Trace (i) is obtained by scanning the LO phase and trace (iii) by locking to the squeezed amplitude

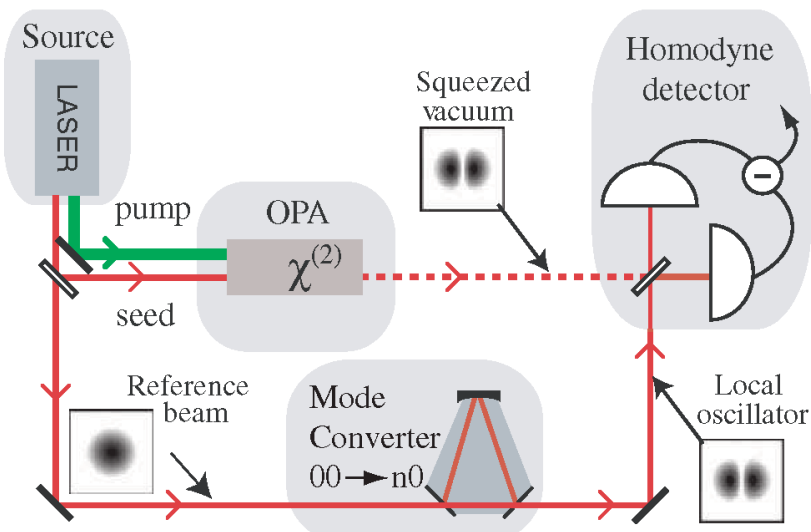

FIG. 3 (color). Experimental setup to generate TEM $_{n 0}$ transverse mode squeezing. An OPA is seeded with an infrared misaligned $\mathrm{TEM}_{00}$ beam. The cavity is then locked to the $\mathrm{TEM}_{n 0}$ mode and pumped with a $\mathrm{SH} \mathrm{TEM}_{00}$ beam. The $\mathrm{TEM}_{n 0}$ squeezed beam thereby produced is analyzed using spatial homodyne detection, whose TEM $_{n 0}$ LO is created from a misaligned ring cavity. Transverse beam profiles are represented in the case of $\mathrm{TEM}_{10}$ mode squeezing. 


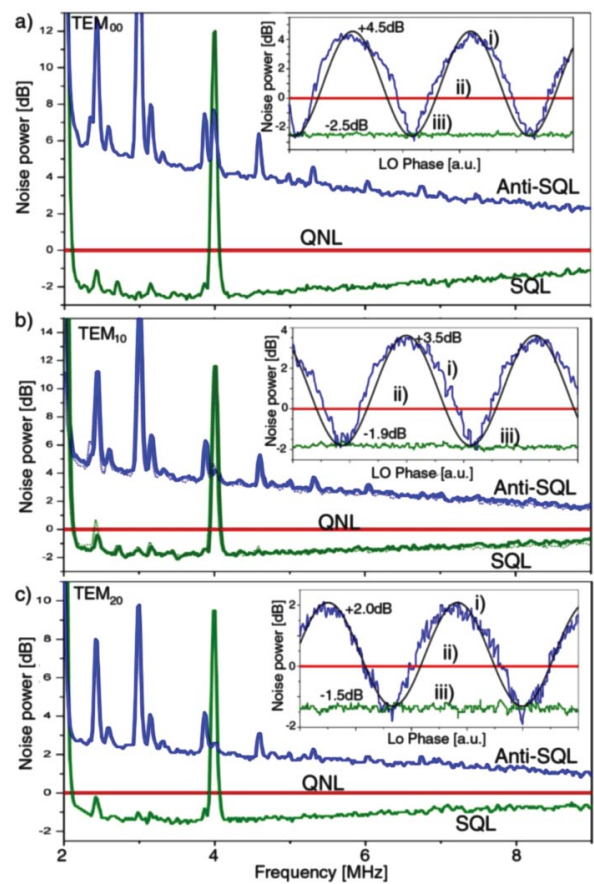

FIG. 4 (color). Squeezing and antisqueezing spectra measured in the (a) $\mathrm{TEM}_{00}$, (b) $\mathrm{TEM}_{10}$, (c) $\mathrm{TEM}_{20}$ modes. All traces are taken with a resolution bandwidth of $30 \mathrm{kHz}$ and a video bandwidth of 30, over more than $1 \mathrm{~min}$. For (b), we also present the results obtained with a coherent copropagating TEM $_{00}$ mode. The insets show the noise properties at $4.45 \mathrm{MHz}$, normalized to the QNL (ii), when the LO phase is (i) scanned, or (iii) locked to the squeezing quadrature.

quadrature. We measured squeezing and antisqueezing values of $-2.5 \pm 0.4 \mathrm{~dB} /+4.5 \pm 0.5 \mathrm{~dB}$ for the $\mathrm{TEM}_{00},-1.9 \pm 0.3 \mathrm{~dB} /+3.5 \pm 0.4 \mathrm{~dB}$ for the $\mathrm{TEM}_{10}$, and $-1.5 \pm 0.3 \mathrm{~dB} /+2.0 \pm 0.4 \mathrm{~dB}$ for the $\mathrm{TEM}_{20}$ mode. From Fig. 4 can be seen that our squeezed states are almost pure states, which indicates that the losses are much smaller then the squeezed states presented in Ref. [30].

We have checked that the detector shows squeezing only when both LO and resonant mode inside the OPA are set to the same mode. To prove the feasibility of efficient combination of such nonclassical fields, we have investigated for any parasite crosstalk between two orthogonal modes. As an example, we have compared the nonclassical properties of a multimode beam, namely, a TEM 10 squeezed mode, with and without the presence of a coherent $\mathrm{TEM}_{00}$ copropagating beam. The great similarity of the spectra shown in Fig. 4(b) clearly demonstrates that the TEM $_{00}$ beam does not modify the squeezing and antisqueezing of the $\mathrm{TEM}_{10}$ mode, which is a formal proof that no parasite crosstalk exists between orthogonal modes.

In summary, our experiments have demonstrated the key components for multimode parallel quantum information systems with $\mathrm{H}-\mathrm{G}$ modes. The encoding of quantum information is direct, efficient, and independent since the higher order modes are exactly linked to the position, momentum, size, and focussing of a $\mathrm{TEM}_{00}$ mode. The quality of detection of the squeezing in each spatial mode shows that it is now practical to synthesize a multimode beam by using our established techniques for lossless mode combination and analysis (Fig. 1). These results open the way for quantum information processing with continuous variables in the transverse plane. The technology for multiple squeezers driven by one laser is available [32], and we can now consider the synthesis of multimode beams with at least five orthogonal modes, each with a pair of quantum variables (Fig. 1). We are on the way to generate spatially entangled beams [33] and test the original EPR proposal. We envision information protocols that are based on parallel mode processing and the future challenge is to design such protocols.

We thank M.T.L. Hsu, G. Hétet, O. Glöckl, and R. Senior for important discussions. This work was supported by the Australian Research Council Centre of Excellence scheme. M. L. is supported by the Danish Technical Research Council (STVF Project No. 26-03-0304).

*Electronic address: C.Harb@adfa.edu.au

[1] P. Zoller et al., Eur. Phys. J. D 36, 203 (2005).

[2] Z. Y. Ou et al., Phys. Rev. Lett. 68, 3663 (1992).

[3] Ch. Silberhorn et al., Phys. Rev. Lett. 86, 4267 (2001).

[4] C. Schori et al., Phys. Rev. A 66, 033802 (2002).

[5] W.P. Bowen et al., Phys. Rev. A 69, 012304 (2004).

[6] K. McKenzie et al., Phys. Rev. Lett. 93, 161105 (2004).

[7] H. Vahlbruch et al., Phys. Rev. Lett. 95, 211102 (2005).

[8] A. Furusawa et al., Science 282, 706 (1998).

[9] J. Jing et al., Phys. Rev. Lett. 90, 167903 (2003).

[10] A. M. Lance et al., Phys. Rev. Lett. 92, 177903 (2004).

[11] V. Josse et al., Phys. Rev. Lett. 96, 163602 (2006).

[12] S. L. Braunstein et al., Rev. Mod. Phys. 77, 513 (2005).

[13] H.-A. Bachor and T. C. Ralph, A Guide to Experiments in Quantum Optics (Wiley, Berlin, 2004).

[14] L. A. Lugiato et al., J. Opt. B 4, S176 (2002).

[15] C. Schwob et al., Appl. Phys. B 66, 685 (1998).

[16] S. Gigan et al., J. Mod. Opt. 53, 809 (2006).

[17] N. Treps et al., Science 301, 940 (2003).

[18] V. Delaubert et al., quant-ph/0609188.

[19] M. Kolobov et al., Opt. Lett. 85, 3789 (2000).

[20] I. V. Sokolov et al., Opt. Commun. 193, 175 (2001).

[21] C. M. Caves et al., Rev. Mod. Phys. 66, 481 (1994).

[22] A. Zeilinger et al., Science 289, 405 (2000).

[23] N. K. Langford et al., Phys. Rev. Lett. 93, 053601 (2004).

[24] H. Kogelnik, "Coupling and Conversion Coefficients for Optical Modes," in Proceedings of the Symposium on Quasi-Optics, Brooklyn, New York, 1964.

[25] M. T. L. Hsu et al., J. Opt. B 6, 495 (2004).

[26] V. Delaubert et al., Opt. Lett. 31, 1537 (2006).

[27] L. A. Wu et al., Phys. Rev. Lett. 57, 2520 (1986).

[28] P. K. Lam et al., J. Opt. B 1, 469 (1999).

[29] S. Suzuki et al., Appl. Phys. Lett. 89, 061116 (2006).

[30] M. Lassen et al., J. Eur. Opt. Soc. 1, 3 (2006).

[31] A. Vaziri et al., J. Opt. B 4, S47 (2002).

[32] N. Takei et al., Phys. Rev. A 72, 042304 (2005).

[33] M. T. L. Hsu et al., Phys. Rev. A 72, 013802 (2005). 